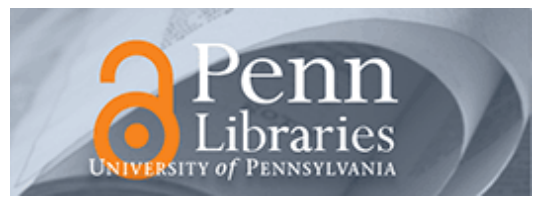

University of Pennsylvania

ScholarlyCommons

Marketing Papers

Wharton Faculty Research

$12-2010$

\title{
The Effect of Accuracy Motivation on Anchoring and Adjustment: Do People Adjust from Provided Anchors?
}

Joseph P. Simmons

University of Pennsylvania

Robyn A. LeBoeuf

Leif D. Nelson

Follow this and additional works at: https://repository.upenn.edu/marketing_papers

Part of the Cognition and Perception Commons, Cognitive Psychology Commons, and the Marketing Commons

\section{Recommended Citation}

Simmons, J. P., LeBoeuf, R. A., \& Nelson, L. D. (2010). The Effect of Accuracy Motivation on Anchoring and Adjustment: Do People Adjust from Provided Anchors?. Journal of Personality and Social Psychology, 99 (6), 917-932. http://dx.doi.org/10.1037/a0021540

This paper is posted at ScholarlyCommons. https://repository.upenn.edu/marketing_papers/317

For more information, please contact repository@pobox.upenn.edu. 


\title{
The Effect of Accuracy Motivation on Anchoring and Adjustment: Do People Adjust from Provided Anchors?
}

\author{
Abstract \\ Increasing accuracy motivation (e.g., by providing monetary incentives for accuracy) often fails to \\ increase adjustment away from provided anchors, a result that has led researchers to conclude that \\ people do not effortfully adjust away from such anchors. We challenge this conclusion. First, we show \\ that people are typically uncertain about which way to adjust from provided anchors and that this \\ uncertainty often causes people to believe that they have initially adjusted too far away from such \\ anchors (Studies 1a and 1b). Then, we show that although accuracy motivation fails to increase the gap \\ between anchors and final estimates when people are uncertain about the direction of adjustment, \\ accuracy motivation does increase anchor-estimate gaps when people are certain about the direction of \\ adjustment, and that this is true regardless of whether the anchors are provided or self-generated \\ (Studies 2, 3a, 3b, and 5). These results suggest that people do effortfully adjust away from provided \\ anchors but that uncertainty about the direction of adjustment makes that adjustment harder to detect \\ than previously assumed. This conclusion has important theoretical implications, suggesting that \\ currently emphasized distinctions between anchor types (self-generated vs. provided) are not \\ fundamental and that ostensibly competing theories of anchoring (selective accessibility and anchoring- \\ and-adjustment) are complementary.
}

\section{Keywords}

debiasing, dual process theories, incentives, inferential correction, intuitive judgment

Disciplines

Business | Cognition and Perception | Cognitive Psychology | Marketing 
The Effect of Accuracy Motivation on Anchoring and Adjustment:

Do People Adjust from Provided Anchors?

$\begin{array}{ccc}\text { Joseph P. Simmons } & \text { Robyn A. LeBoeuf } & \text { Leif D. Nelson } \\ \text { Yale University } & \text { University of Florida } & \text { University of California, } \\ & \text { Berkeley }\end{array}$

In press at the Journal of Personality and Social Psychology

Joseph P. Simmons, Department of Marketing, Yale School of Management; Robyn A. LeBoeuf, Department of Marketing, Warrington College of Business, University of Florida; Leif D. Nelson, Department of Marketing, Haas School of Business, University of California, Berkeley. Portions of this research were presented at the Society for Judgment and Decision Making conference in Houston, Texas (November, 2006) and at the Society for Personality and Social Psychology in Albuquerque, New Mexico (February, 2008). We thank Nick Epley, Justin Kruger, and Mike Norton for thoughtfully commenting on a previous draft of this manuscript, we thank Jeff Galak for his help with Study 3a, and we thank Andrew Meyer and Hannah Perfecto for their help with Studies 3b and 5. Correspondence concerning this manuscript should be addressed to Joseph Simmons, Yale School of Management, 135 Prospect Street, New Haven, Connecticut, 06520, e-mail: joseph.simmons@yale.edu. 


\begin{abstract}
Increasing accuracy motivation (e.g., by providing monetary incentives for accuracy) often fails to increase adjustment away from provided anchors, a result that has led researchers to conclude that people do not effortfully adjust away from such anchors. We challenge this conclusion. First, we show that people are typically uncertain about which way to adjust from provided anchors, and that this uncertainty often causes people to believe that they have initially adjusted too far away from such anchors (Studies 1a and 1b). Then, we show that although accuracy motivation fails to increase the gap between anchors and final estimates when people are uncertain about the direction of adjustment, accuracy motivation does increase anchor-estimate gaps when people are certain about the direction of adjustment, and that this is true regardless of whether the anchors are provided or self-generated (Studies 2, 3a, 3b, and 5). These results suggest that people do effortfully adjust away from provided anchors, but that uncertainty about the direction of adjustment makes that adjustment harder to detect than previously assumed. This conclusion has important theoretical implications, suggesting that currently emphasized distinctions between anchor types (self-generated vs. provided) are not fundamental, and that ostensibly competing theories of anchoring (selective accessibility and anchoring-andadjustment) are complementary.
\end{abstract}


The Effect of Accuracy Motivation on Anchoring and Adjustment:

Do People Adjust from Provided Anchors?

Considering irrelevant values can influence people's estimates of unknown quantities. This fact is known as anchoring and it is arguably one of the most important truths about human judgment (e.g., Gilbert, 2002; Epley \& Gilovich, 2004, 2006; Jacowitz \& Kahneman, 1995; Tversky \& Kahneman, 1974). Anchoring was most famously demonstrated by Tversky and Kahneman (1974), who found that people estimated a greater percentage of African countries in the United Nations after considering a randomly generated "anchor" of 65\% than after considering an anchor of $10 \%$. Researchers have since shown that anchoring arises not only for such general knowledge questions, but also for arguably more consequential judgments, such as buying and selling prices (Carlson, 1990; Green, Jacowitz, Kahneman, \& McFadden, 1998; Simonson \& Drolet, 2004), purchase quantity decisions (Wansink, Kent, \& Hoch, 1998), credit card repayments (Stewart, 2009), negotiation outcomes (Galinsky \& Mussweiler, 2001), appraisals of real estate (Northcraft \& Neale, 1987), personal injury verdicts (Chapman \& Bornstein, 1996), and criminal sentences by legal experts (Englich, Mussweiler, \& Strack, 2006).

Much research has investigated how anchors affect judgment. This research has produced different theories of anchoring and a debate about which theory is correct. This debate has seemingly been resolved by distinguishing two types of anchors: anchors that are provided by an external source (the usual case in numerical anchoring experiments) and anchors that are selfgenerated (Epley \& Gilovich, 2001, 2004, 2005, 2006). Researchers now accept that these different anchor types induce different psychological processes, and that distinct theories are needed to explain how self-generated and provided anchors affect judgment (Epley \& Gilovich, 2006). Our goal is to argue that this distinction is unnecessary, that provided and self-generated 
anchors affect judgment through largely similar processes, and that people do effortfully adjust from both provided and self-generated anchors. We accomplish this by investigating the effect of accuracy motivation on anchoring, an effect on which major theoretical distinctions hinge.

\section{Theories of Anchoring}

Anchoring-and-Adjustment Theory

Anchoring-and-adjustment is the traditional explanation of how anchors affect judgment. According to this theory, the process of generating estimates after considering anchor values proceeds in multiple stages (Epley \& Gilovich, 2001, 2004, 2006; Epley, Keysar, Van Boven, \& Gilovich, 2004; Quattrone, 1982; Tversky \& Kahneman, 1974). Consider the typical anchoring study, in which people estimate some quantity (e.g., the length of the Mississippi River) after first assessing whether the quantity is greater or less than a provided anchor value (e.g., 1200 miles). According to anchoring-and-adjustment theory, estimate generation works as follows (see Figure 1a). First, people decide whether the correct value is greater or less than the anchor. Then, they adjust from the anchor by generating an initial value. Next, people test whether this value seems reasonable or whether they should adjust their estimate again. People who consider their initial estimate to be "good enough" will cease adjustment and deliver that estimate. In contrast, people who consider their initial estimate to require modification will adjust their estimate further away from the anchor value. People repeat the process of testing and adjusting until they are ultimately satisfied with their estimate. Anchoring effects are thought to arise partly because people are often not motivated to extensively revise their estimates, and partly because most people consider a wide range of values to be plausible estimates. Adjustments thus tend to be insufficient, with people settling on a plausible value that is relatively close to the anchor (Epley \& Gilovich, 2006). 
An important prediction of anchoring-and-adjustment theory concerns the effect of motivation on adjustment. Relative to unmotivated people, people who are motivated to be accurate (e.g., because they have a monetary incentive to give correct answers) should have higher standards, should be less likely to think that their estimates are "good enough,” and should therefore be more likely to adjust extensively. Thus, motivated individuals’ final estimates should be further away from anchor values, and motivation should decrease anchoring effects. However, this prediction has been contradicted by decades of research showing that increased accuracy motivation fails to reduce anchoring in the typical paradigm (Chapman \& Johnson, 2002; Epley \& Gilovich, 2005; Tversky \& Kahneman, 1974; but see Wright \& Anderson, 1989, for a marginally significant exception). Indeed, in their review of the anchoring literature, Chapman and Johnson (2002) concluded that "incentives reduce anchoring very little if at all” (p. 125). Because of this null effect of motivation, many researchers have rejected anchoring-and-adjustment theory and concluded that people do not effortfully adjust from provided anchors (Chapman \& Johnson, 2002; Epley \& Gilovich, 2005, 2006; Strack \& Mussweiler, 1997).

\section{Selective Accessibility Model}

While researchers were souring on anchoring-and-adjustment theory, Strack and Mussweiler (1997) proposed a different explanation of anchoring. According to their selective accessibility model, anchors prompt people to test the hypothesis that the true value is equal to the anchor value. Because testing hypotheses increases the accessibility of hypothesis-consistent information (e.g., Klayman \& Ha, 1987; Wason, 1960), testing whether the true value is equal to the anchor should increase the accessibility of anchor-consistent information. The selective accessibility model posits that people use this accessible anchor-consistent information when 
generating their estimates, and that this produces anchoring effects. Thus, merely considering an anchor of 1200 miles brings to mind information suggesting that the Mississippi River’s length may be near that value; use of such anchor-consistent accessible information - rather than a process of (insufficient) adjustment - is thought to lead people to generate an estimate that is close to the anchor.

This account draws power from its ability to make unique predictions, many of which have empirical support (Chapman \& Johnson, 2002; Epley, 2004; Mussweiler \& Strack, 1999, 2000; Strack \& Mussweiler, 1997). Most important for this article, and in contrast to anchoring-andadjustment theory, the selective accessibility model posits no adjustment process, and is therefore not undermined by the null effects of motivation on anchoring reported in the literature. Thus, there seems to be good reason for favoring the selective accessibility model and for disfavoring anchoring-and-adjustment theory as an explanation of how anchors affect judgment. Self-Generated vs. Provided Anchors

Although anchoring-and-adjustment theory has earned disfavor as a description of traditional anchoring effects, it has made a comeback as an explanation of anchoring effects in a different paradigm. In an important line of research, Epley and Gilovich (2001, 2004, 2005, 2006) have suggested that although the selective accessibility model persuasively describes how anchors affect judgment when the anchors are provided by the experimenter, anchoring-and-adjustment theory describes how anchors affect judgment when the anchors are self-generated. To illustrate the self-generated type, consider a participant who is asked to estimate the year that George Washington became President of the United States. Although the participant may not know the true answer to this question, she may think of an anchor she knows to be lower or higher (e.g., “The Declaration of Independence was signed in 1776, so it must be after that”), and then adjust 
in what she believes to be the correct direction (e.g., 1777, 1779, and so on). In this case, the anchor (1776) is self-generated and Epley and Gilovich argue that people adjust from such anchors.

In support of this claim, Epley and Gilovich (2001, 2004, 2005, 2006) have presented evidence suggesting that self-generated and provided anchors operate differently. Most critically, increasing accuracy motivation increases adjustment away from self-generated but not provided anchors, seemingly implicating effortful adjustment as a process underlying self-generated, but not provided, anchoring effects (Epley \& Gilovich, 2005). On the basis of this differential effect of motivation, Epley and Gilovich (2006, p. 316) have concluded that it is "clear that not all anchoring effects result from the same psychological mechanism” and that "anchoring effects observed in the standard anchoring paradigm . . . are the result of an enhanced accessibility of anchor-consistent information, not insufficient adjustment.” That is, although anchoring-andadjustment theory seems to best explain how self-generated anchors affect judgment, the selective accessibility model may best explain how provided anchors affect judgment (see Figure 2a). This conclusion is now widely accepted (e.g., Chapman \& Johnson, 2002; Epley, 2004; Epley \& Gilovich, 2006; Mussweiler \& Englich, 2005; Simonson \& Drolet, 2004).

A Revised Theory of Anchoring and Adjustment

As this review makes clear, anchoring theorizing currently hinges on the null effect of motivation on adjustment from provided anchors, as researchers assume that this result indicates that provided anchors do not induce adjustment processes.

In contrast, we suggest that people effortfully adjust from all anchors, regardless of whether they are externally provided or self-generated. We suggest that confusion on this point has arisen because of three misconceptions in the literature. First, most anchoring theorists treat selective 
accessibility and anchoring-and-adjustment as competing explanations of anchoring, therefore assuming that evidence favoring selective accessibility constitutes evidence against anchoringand-adjustment (and vice versa). However, selective accessibility and anchoring-and-adjustment are not logically contradictory theories: Showing that people selectively recruit anchor-consistent information does not rule out the possibility that people also effortfully adjust from anchor values. Thus, the fact that data support the selective accessibility model's account of anchoring when anchors are provided does not, in and of itself, mean that people fail to effortfully adjust from provided anchors. Rather, on purely logical grounds, both selective accessibility and adjustment could contribute to any given anchoring effect (see Figure 2b).

Second, although anchoring-and-adjustment theory currently assumes that people who are motivated to correct their initial estimates will always adjust by generating estimates that are further away from the anchor (Figure 1a; Epley \& Gilovich, 2006), we suggest that corrections of initial estimates may - and do - occur in both directions. Although people who believe that their initial estimates are too close to the anchor will indeed correct their estimates by adjusting even further away from the anchor, people may sometimes believe that their initial estimates are too far from the anchor, and they will correct their estimates by adjusting toward the anchor when motivated to be accurate (cf. Wegener \& Petty, 1995).

Third, as a consequence, increased motivation should not uniformly increase adjustment away from anchors. Rather, it should only increase the distance between anchors and final estimates (hereafter referred to as anchor-estimate gaps) when people believe that their initial estimates are too close to the anchor (i.e., when people believe that their initial adjustments were insufficient). When people instead believe that their initial estimates are too far from the anchor (i.e., when people believe that their initial adjustments were too extreme), then increased 
motivation should produce final estimates that are closer to anchors. By this account, anchoringand-adjustment need not - and should not - predict that increasing accuracy motivation will always increase anchor-estimate gaps (and decrease anchoring effects). Instead, anchoring-andadjustment theory must predict that the effect of motivation depends on people's beliefs about whether they initially over- or under-adjusted.

To test this revised view of adjustment, we must first consider what determines whether people believe that they have adjusted insufficiently (versus too far) from anchors. We suggest that one important determinant of this belief is whether people are certain about in which direction to adjust from the anchors in the first place. In particular, we suggest that people will be more likely to believe that they have adjusted insufficiently from an anchor when they are certain about the correct direction of adjustment than when they are uncertain.

To understand the motivation for this prediction, imagine trying to estimate the average number of hairs on a buffalo after encountering an anchor of 500,000. Knowing virtually nothing about the topic (except that buffaloes are hairy creatures), you might venture an uncertain guess that the average number is greater than 500,000, and so you might estimate 520,000 . Imagine that you then learn that you will be paid based on how close your estimate is to the correct answer, and that you can revise your estimate to be more accurate. Although current anchoringand-adjustment theory expects you to adjust even further away from the anchor now that you are motivated to be accurate, this expectation assumes that you believe that you adjusted insufficiently (i.e., that you believe that 520,000 is below the true value). However, given how much uncertainty you had about the correct direction in which to adjust in the first place, you may instead worry that you adjusted too far, or in the wrong direction altogether. Thus, in the face of a newfound motivation to be accurate, you may decide to stick with your original 
estimate, or you may actually adjust your estimate toward the anchor rather than further away from it (see Figure 1b).

Thus, when people are unsure if their initial adjustments are in the correct direction, we expect them to often believe that they have initially adjusted sufficiently or too far, and to be unlikely to adjust their estimates further away from the anchor when motivated to be accurate. In contrast, when people are certain about which direction to adjust from anchor values (e.g., when they are asked to estimate whether the average number of hairs on a buffalo exceeds 10), they need not worry about having adjusted in the wrong direction. Indeed, they may infer from their certainty about the adjustment direction that the correct answer is quite far from the anchor (cf. Simmons \& Nelson, 2006), and they may often believe that they have adjusted insufficiently. Thus, compared to those who are uncertain about the direction of adjustment, people who are certain may be more likely to believe that they have adjusted insufficiently, and more likely to adjust their initial estimates further away from the anchor when motivated to be accurate.

To summarize, we make the following predictions:

1. People will be more likely to believe that they have insufficiently adjusted from anchor values when they are certain about the direction of adjustment than when they are uncertain about the direction of adjustment.

2. Increasing accuracy motivation will be more likely to increase anchor-estimate gaps for people who are certain about the direction of adjustment than for people who are uncertain about the direction of adjustment (because those who are certain will be more likely to believe they have adjusted insufficiently).

The Current Research 
Our studies explore these hypotheses, and in so doing, aim to shed new light on motivation's influence on anchoring, and specifically on the pivotal finding that motivation tends to increase adjustment away from self-generated anchors but not from provided anchors (Epley \& Gilovich, 2005). We suggest that motivation has previously been shown to have different effects for these anchor types not because those types induce different psychological processes, but because they induce differences in certainty about the direction of adjustment. Although people are often uncertain about the direction in which to adjust from provided anchors (e.g., Jacowitz \& Kahneman, 1995) and thus, according to our framework, may not respond to additional motivation by adjusting further, people tend to choose self-generated anchors precisely because such anchors confer certainty about the direction of adjustment (Epley \& Gilovich, 2001): Participants who estimate George Washington's election year by starting with an anchor of 1776 likely do so precisely because they know that the true answer must exceed the anchor. Such participants, according to our framework, may thus be relatively more likely to believe that their initial adjustments were insufficient and that further adjustment is warranted.

If differences in adjustment-direction certainty explain the distinction between self-generated and provided anchors, then provided anchors should "behave” like self-generated anchors when people know in which direction to adjust from such anchors, and self-generated anchors should "behave” like provided anchors when people do not know in which direction to adjust from such anchors. Thus, although previous research has shown that accuracy motivation increases anchorestimate gaps for self-generated anchors only (Epley \& Gilovich, 2005), we should observe that accuracy motivation increases anchor-estimate gaps for provided anchors whenever participants are certain about the direction of adjustment from those anchors. Similarly, although previous research suggests that accuracy motivation increases anchor-estimate gaps for all self-generated 
anchors, we should observe that this effect fails to obtain whenever participants are uncertain about the direction of adjustment from those anchors.

In Study 1, we begin by testing our hypothesis that certainty about the direction of adjustment affects people's beliefs about whether they initially adjusted insufficiently (vs. too far) from an anchor. In Studies 2 and 3, we then investigate whether certainty about the direction of adjustment also determines whether motivation will increase the gap between provided anchors and final estimates. Although the vast majority of studies investigating the effects of motivation on adjustment from provided anchors have reported null effects, we predict that motivation will increase gaps between estimates and provided anchors as long as people are certain about the correct direction of adjustment.

Studies 4 and 5 more directly examine whether the reported differences in the literature between self-generated and provided anchors can be explained by differences in adjustmentdirection certainty: Study 4 investigates whether self-generated anchors inspire more adjustment-direction certainty than provided anchors, and Study 5 investigates whether motivation's effect on the gap between self-generated anchors and final estimates depends on whether people are certain (vs. uncertain) about which way to adjust from such anchors. Although motivation has previously been found to increase anchor-estimate gaps for selfgenerated anchors (Epley \& Gilovich, 2005), we predict that motivation will fail to increase gaps between estimates and self-generated anchors when people are uncertain about the correct direction of adjustment.

Studies 1a and 1b: Beliefs about the Sufficiency of Adjustment

Studies $1 \mathrm{a}$ and $1 \mathrm{~b}$ investigated our initial proposition that people will be more likely to believe that they have insufficiently adjusted from an anchor when they are certain about the 
direction of adjustment from that anchor. In both studies, participants answered generalknowledge questions after encountering provided anchors. We manipulated whether or not people knew the direction of adjustment, and we assessed their beliefs about the sufficiency of their adjustments. We expected participants who knew the direction of adjustment to be more likely to believe that their adjustments were insufficient than participants who did not know the direction of adjustment.

\section{Study la Method}

Participants. One hundred seventy-nine undergraduates at two private universities participated for payment.

Procedure. During a laboratory session, participants answered two general-knowledge questions that provided anchors. The first question asked them to estimate the distance between Detroit, Michigan and Phoenix, Arizona from an anchor of 1200 miles, and the second question asked them to estimate the population of Colorado from an anchor of 10 million people.

Before they estimated the correct answer, participants in the direction-known condition were told, for each question, whether the correct answer was above or below the anchor value. Participants in the direction-unknown condition were not given this information for either question. For example, participants in the direction-known condition began by reading the assertion, "It is true that the distance between Detroit, Michigan and Phoenix, Arizona is less than 1200 miles,” whereas those in the direction-unknown condition began by answering the question, "Is the distance between Detroit, Michigan and Phoenix, Arizona greater or less than 1200 miles?” (Note that the direction-unknown condition followed the procedure typically used in provided-anchor studies). 
For each question, participants (1) estimated the correct answer and subsequently (2) indicated whether they believed that their estimate, if not exactly correct, was above or below the true value. For example, after participants estimated the population of Colorado, they were asked, "Assuming that your previous estimate is not exactly correct, do you think that your estimate is above or below the exact population of Colorado?’ Belief in insufficient adjustment was presumed when participants (1) adjusted upward from an anchor and indicated that their estimate was below the true value, or (2) adjusted downward from an anchor and indicated that their estimate was above the true value. Belief in over-adjustment was presumed when participants (1) adjusted upward from an anchor and indicated that their estimate was above the true value, or (2) adjusted downward from an anchor and indicated that their estimate was below the true value.

\section{Study la Results and Discussion}

We excluded $4.2 \%$ of estimates because they were missing, imprecise (e.g., "900-920 miles”), or equal to the provided anchor value.

Figure 3 shows the results. For the Detroit-Phoenix item, only 27\% of participants believed that their adjustments were insufficient when the direction of adjustment was unknown, but this proportion was significantly higher when the direction of adjustment was specified (51\%), $\chi^{2}(1$, $N=170)=10.69, p=.001$. We observed a similar result for the Colorado item, where specifying the direction of adjustment increased the proportion believing they had insufficiently adjusted from $31 \%$ to $48 \%, \chi^{2}(1, N=173)=6.32, p=.01$.

Thus, the results of this study suggest that when the direction of adjustment is unknown (as in the standard anchoring paradigm), people may often assume that their initial adjustments from provided anchors are too extreme rather than insufficient. This finding is important, as it 
challenges the assumption (of traditional anchoring-and-adjustment theory) that increased accuracy motivation should always cause people to move further away from an anchor. ${ }^{1}$ Study 1b sought to replicate these results using a different manipulation of adjustment certainty.

\section{Study $1 b$ Method}

Participants. One hundred ninety-eight undergraduates at a public university participated for course credit.

Procedure. As in Study 1a, participants answered two general-knowledge questions that provided anchors. The first question asked them to estimate the average high temperature in Miami, Florida in the month of August and the second question asked them to estimate the age of actor/comedian Jerry Seinfeld. As in Study 1a, for each question, participants (1) estimated the correct answer and subsequently (2) indicated whether they believed that their estimate, if not exactly correct, was above or below the true value. In addition, to discourage participants from assuming that the anchors were informative (Grice, 1975; Schwarz, 1996), they were told that the anchors were uninformative, and that the "true value may be close to OR far from” the anchor.

Studies 1a and 1b differed in the manipulation of certainty about the direction of adjustment. Whereas Study 1a's manipulation involved simply telling some participants the correct direction of adjustment, Study 1b manipulated adjustment-direction certainty by manipulating whether each anchor value was plausible or obviously implausible. For the Miami question, the plausible anchor was 86 degrees Fahrenheit and the implausible anchor was 10 degrees Fahrenheit. For the Seinfeld question, the plausible anchor was 47 years and the implausible anchor was 18 years. In all cases, participants were first asked to indicate whether the true value was higher or lower than the anchor, and then to estimate the true value. We expected participants to be certain about 
which direction to adjust from implausible anchors (e.g., it is obvious that Miami’s average high temperature in August exceeds 10 degrees) but not from plausible anchors (e.g., it is less obvious whether that temperature exceeds 86 degrees). We orthogonally manipulated the plausibility of each item’s anchor.

\section{Study 1 b Results and Discussion}

We excluded $2.8 \%$ of estimates that were missing or equal to the provided anchor value.

Figure 3 shows the results. For the Miami question, only $41 \%$ of participants believed that their adjustments were insufficient when the anchor was plausible and the direction of adjustment was therefore uncertain. However, when the anchor was implausible, and the direction of adjustment was therefore certain, more than half of the participants (60\%) believed that their adjustments were insufficient. The difference between conditions was significant, $\chi^{2}(1$, $N=194)=6.68, p=.01$. We observed a similar result for the Seinfeld question: The proportion believing they had insufficiently adjusted was greater when the anchor was implausible (55\%) than when it was plausible (40\%), $\chi^{2}(1, N=191)=4.49, p<.04$.

The results of Studies 1a and 1b are consistent with each other and with our theorizing. First, they show that, contrary to prior assumptions, people tend to believe that they have over-adjusted from provided anchors when, as is typically the case, the direction of adjustment is not obvious. This supports our suggestion that motivation usually fails to increase gaps between estimates and provided anchors because people often believe that they have initially adjusted too far from these anchors. Second, the results show that the belief that one has insufficiently adjusted is more likely to arise when people are certain about the direction of adjustment. Thus, imparting participants with certainty about the adjustment direction should, by increasing their tendency to believe that their initial adjustments are insufficient, create a more favorable context for 
motivation to increase anchor-estimate gaps. (And, of course, motivation should do this if people are indeed adjusting from provided anchors). Studies 2 and 3 investigated this possibility.

Study 2: Motivation Increases Anchor-Estimate Gaps When the Direction of Adjustment from Provided Anchors is Known

As reviewed in the introduction, most studies have shown that accuracy motivation fails to increase adjustment away from provided anchors. Largely because of this, most researchers have concluded that people do not effortfully adjust from these anchors. However, Study 1 suggested a different reason for the null effects of motivation: Participants may often be unsure of which direction to adjust from provided anchors, and thus may often believe that their initial adjustments are too extreme. Thus, they may not consider it wise to adjust further away from anchors when motivated to be accurate.

In Study 2, we sought to build on Study 1's findings and to demonstrate that motivation does increase anchor-estimate gaps as long as participants are certain about the direction of adjustment. Toward this aim, we asked participants to answer questions that provided anchors. We manipulated whether participants were motivated to be accurate and whether they were told the correct direction of adjustment. We expected motivation to increase anchor-estimate gaps when participants knew in which direction to adjust.

\section{Method}

Participants. Two hundred forty-nine undergraduates from a public university participated for course credit.

Procedure. As part of a laboratory session, participants answered 10 general-knowledge questions that each provided anchors (Table 1 displays the items). As in Study 1b, we included an instruction designed to discourage participants from assuming that the anchors were 
informative. Across participants, we manipulated three variables. First, as in Study 1a, we manipulated whether the correct direction of adjustment from each anchor was known (e.g., “The length of the Mississippi River is greater than 1200 miles.”) or unknown (e.g., "Is the length of the Mississippi River greater or less than 1200 miles?”). Second, we manipulated whether participants were motivated to be accurate. In the motivated condition, participants began by reading, "In this questionnaire, you have the opportunity to WIN \$100!!” They were told that we would “award \$100 to the person who provides the most accurate estimates overall.” These participants then reported their e-mail addresses so we could contact them if they won. In contrast, participants in the unmotivated condition were not offered the opportunity to win the $\$ 100$ prize and were not asked to provide e-mail addresses. Finally, we manipulated anchor values by creating two versions of the questionnaire. Anchors that were higher than the correct answer in one version were lower than the correct answer in the other version, and vice versa. For example, the Mississippi River item used an anchor of 1200 miles in one version and 3500 miles in the other version (see Table 1). Each version contained a mix of high and low anchors.

\section{Results and Discussion}

We excluded $1.8 \%$ of responses because they were missing, illegible, or imprecise.

To conduct the critical analysis, we computed each participant's average anchor-estimate gap using methods similar to Epley and Gilovich (2001, 2004). Specifically, we (1) computed the absolute value of the difference between each estimate and the anchor, (2) z-scored these values separately for each question and for each anchor value, and (3) averaged, for each participant, the z-scored values across all questions. Higher positive numbers indicate a larger gap between anchors and final estimates. 
A Direction (Known vs. Unknown) x Motivation (Motivated vs. Unmotivated) betweensubjects ANOVA on these anchor-estimate gaps yielded only the predicted Direction $\mathrm{x}$ Motivation interaction, $F(1,245)=4.04, p<.05$. As shown in Figure 4, accuracy motivation increased anchor-estimate gaps when the direction of adjustment was known, $t(123)=2.19, p=$ .03 , but not when the direction of adjustment was unknown, $t(122)=-0.55, p=.58$. Thus, motivated participants adjusted further away from the provided anchors, but only when they knew the correct direction of adjustment.

The previous analysis focused on the distance between anchors and final estimates as the target dependent measure, but because we manipulated anchor values in this study, we could also analyze the effect of our manipulations on the size of each item’s anchoring effect. In each Direction x Motivation cell of the design, we computed the anchoring effect for each question based on the formula used by Jacowitz and Kahneman (1995):

(High anchor mean estimate - Low anchor mean estimate)/(High anchor - Low anchor) Thus, a mean estimate of 3000 from an anchor of 3500 and a mean estimate of 1500 from an anchor of 1200 would yield an anchoring effect of $(3000-1500) /(3500-1200)=0.65$. Higher numbers indicate a bigger anchoring effect.

A Direction x Motivation repeated-measures ANOVA on the size of each question's anchoring effect yielded a significant main effect of Direction, $F(1,9)=21.31, p=.001$, as well as a marginally significant interaction, $F(1,9)=4.70, p<.06$. On average, the anchoring effect was bigger when the direction of adjustment was unknown $(M=.51, S E=.06)$ than when it was known $(M=.32, S E=.07)$. This effect may have emerged because anchoring effects are increased by adjustments in the incorrect direction, a tendency that is strongly reduced by telling participants the correct direction in which to adjust. More important, the interaction supported 
our main hypothesis. Increasing accuracy motivation significantly decreased anchoring when the direction of adjustment was known, $t(9)=3.92, p=.003$, and, as shown in Figure 5, this tendency was evident for all 10 items. In contrast, accuracy motivation did not decrease anchoring when the direction of adjustment was unknown, $t(9)=-0.26, p=.80$.

These results show that accuracy motivation can (1) increase the distance between final estimates and provided anchors and, in so doing, (2) decrease the size of anchoring effects, as long as participants know in which direction to adjust from these anchors. Most previous studies found a null effect of motivation on the effects of provided anchors, but Studies 1 and 2 suggest that this null effect arose in large part because participants in prior studies were uncertain about which direction to adjust from the anchors. It may thus have been premature to assert, on the basis of those null effects, that adjustment plays no role in the effects of provided anchors. Indeed, the current results suggest that people $d o$ effortfully adjust from provided anchors and that the amount of adjustment can be increased with accuracy motivation. In Study 3, we attempted to accumulate further support for this notion.

Studies 3a and 3b: Motivation Increases Anchor-Estimate Gaps When Provided Anchors Are Implausible

In Studies 3a and 3b, we again investigated whether the effect of motivation on anchoring depends on whether people know in which direction to adjust, but in these studies we manipulated adjustment-direction certainty differently (and, arguably, more naturally) by altering the plausibility of the anchors. As in Study 1b, we reasoned that, although participants should be uncertain about which way to adjust from plausible anchors, they should know in which direction to adjust from implausible anchors (and, as shown in Study 1b, they should believe that initial adjustments from implausible anchors are insufficient). Hence, we expected accuracy 
motivation to move estimates further away from implausible anchors but not from plausible anchors.

\section{Study 3 a Method}

Participants. One hundred twenty-seven undergraduates at a private university participated for a chance to win a lottery prize and a $\$ 50$ gift certificate.

Procedure. In an online survey, we asked participants to answer eight general-knowledge questions that provided anchors (see Table 2). As in the previous study, we told all participants that the anchors were uninformative.

The study consisted of two stages. In Stage 1, a computer presented the questions one at a time, and participants answered each question by making a direction-of-adjustment decision followed by an estimate, as in the standard anchoring paradigm. For example, participants were asked, “Did the television show Seinfeld first appear on the air before or after 2005?” and then they estimated the year it first appeared on the air. We manipulated the plausibility of each item's anchor between participants, and half of each participant's questions featured implausible anchors (see Table 2). After Stage 1, we introduced Stage 2 (and our motivation manipulation) by telling participants the following:

Now that you have completed all eight items, we are going to give you a chance to revise your estimates. For each question, you will be reminded of your answers, and you will be asked to revise them. You can change all, some, or none of your answers. If your final answer - after the change - is close enough to the true answer, then you will receive a point. Points are VERY important, because the more points you earn in this study, the greater your chances are of winning the grand prize of a \$50 amazon.com gift certificate! ... Thus, it is important that you give a final answer that is as accurate as possible. 
In Stage 2, participants answered Stage 1's questions in the same order. For each question, they were reminded of the original anchor, of their original answer to the direction-of-adjustment question, and of their original estimate. For example, a participant who estimated that Seinfeld first aired in 1992 after considering an anchor of 2005 was told:

You indicated that the television show Seinfeld first appeared on the air before 2005. Your exact estimate of the year that Seinfeld appeared on the air was 1992. You now have a chance to revise this estimate in order to make it more accurate. If you do not wish to revise your estimate, please just type the answer that you gave previously. In which year did Seinfeld first appear on the air?

On each screen in Stage 2, participants were reminded that accurate answers were important for earning a chance to win the $\$ 50$ gift certificate.

Thus, all participants answered the questions first without incentives (Stage 1) and then again with incentives for accuracy (Stage 2). This within-subjects manipulation of incentives allowed us to precisely measure how participants changed their unmotivated estimates once incentives were introduced.

\section{Study 3 a Results and Discussion}

We excluded $1.3 \%$ of responses that were missing or extreme (presumably because of typing errors).

To analyze the effect of motivation on anchor-estimate gaps, we first computed the difference between participants’ unmotivated Stage 1 estimates and their incentive-motivated Stage 2 estimates, and then we converted these differences into standard deviation units (by dividing each difference by the standard deviation of the item's difference scores). This resulted in a motivation score that reflected how far newly-motivated participants moved away from (or 
back toward) the anchor. This score was positive if motivation increased the anchor-estimate gap, negative if motivation decreased the anchor-estimate gap, and zero if the Stage 2 and Stage 1 estimates were identical.

We averaged participants’ motivation scores separately for the four implausible-anchor and plausible-anchor questions. A paired t-test on these scores revealed the predicted effect, $t(126)=$ 4.61, $p<.001$ : Motivation increased anchor-estimate gaps more for implausible anchors $(M=$ $0.26, S E=.06)$ than for plausible anchors $(M=-0.07, S E=.03$; see Figure 6$)$. In fact, motivation reliably increased anchor-estimate gaps for implausible anchors, $t(126)=4.76, p<.001$, but decreased anchor-estimate gaps for plausible anchors, $t(126)=-1.94, p<.055$. $^{2}$ Because people should know in which direction to adjust from implausible anchors, these results are consistent with our contention that motivation increases adjustment from provided anchors, but only when people are certain about the direction of adjustment.

One potential concern with Study 3a is that the within-subjects manipulation of incentives required participants to explicitly consider whether or not to revise their estimates, which may have encouraged them to revise their estimates more frequently (or extensively) than they would have if we had used a between-subjects incentives manipulation. Although this concern cannot explain the difference observed between anchor types in Study 3a (i.e., why incentives caused people to adjust further away from implausible anchors but back toward plausible anchors), we thought it prudent to replicate this result using a between-subjects manipulation of incentives.

\section{Study $3 b$ Method}

Participants. Fifty-seven undergraduates at a private university participated for \$5. 
Procedure. At the beginning of a short experimental session, we asked participants to answer six general-knowledge questions that provided anchors (see Table 3). ${ }^{3}$ As before, we told all participants that the anchors were uninformative.

This study featured a 2 (Plausibility: Implausible vs. Plausible anchors) x 2 (Motivation: Motivated vs. Unmotivated) between-subjects design. Participants in the implausible-anchors condition received implausible anchors for all six items, whereas participants in the plausibleanchors condition received plausible anchors for all six items. Participants in the unmotivated condition were not promised any reward for answering the general knowledge questions carefully, whereas participants in the motivated condition were promised an additional \$1 for each answer they generated that was close to the right answer. Participants in the motivated condition were paid immediately after completing the task.

\section{Study $3 b$ Results and Discussion}

We excluded $2.4 \%$ of responses because they were missing.

Our theory predicts that accuracy motivation will be more likely to increase adjustment further away from implausible anchors than from plausible anchors. To test this, we first computed each participant’s average anchor-estimate gap, as in Study 2. This computation yielded a z-score, with higher numbers indicating a larger distance between anchors and final estimates.

We then conducted a Plausibility (Implausible vs. Plausible) x Motivation (Motivated vs. Unmotivated) between-subjects ANOVA on these anchor-estimate gaps. This analysis yielded only the predicted Plausibility x Motivation interaction, $F(1,53)=6.03, p<.02$. As shown in Figure 7, and consistent with the results of Study 3a, accuracy motivation increased anchorestimate gaps when the anchors were implausible, $t(26)=1.84, p<.04$ (one-tailed), but tended to 
decrease anchor-estimate gaps when the anchors were plausible, $t(27)=-1.62, p<.06$ (onetailed). ${ }^{4}$ Once again this constitutes evidence that accuracy motivation does increase adjustment away from provided anchors, as long as participants are certain about the direction in which to adjust. Although prior findings suggested that motivation had no effects for provided anchors, our results suggest that those null effects may have arisen because participants were uncertain about which direction to adjust from those anchors, and not because participants were not adjusting at all.

Study 4: Greater Certainty in the Direction of Adjustment from Self-Generated Anchors Than From Provided Anchors

Studies 1 through 3 showed that accuracy motivation can increase adjustment away from provided anchors as long as participants are certain about the direction in which to adjust from those anchors. Recall that researchers have typically found that motivation increases adjustment away from self-generated anchors but not from provided anchors (e.g., Epley \& Gilovich, 2005). We argue that differences in adjustment-direction certainty may explain this pattern of results, and that one key difference between self-generated and provided anchors is that people are generally more certain about the direction of adjustment from the former than the latter. We thus argue that this difference in adjustment-direction certainty (rather than some fundamental difference between the anchors or the types of processing that they engender) accounts for the previously observed differential impact of motivation. Study 4 investigates whether people are, in fact, typically more certain about the direction of adjustment when anchors are self-generated than when they are provided.

\section{Method}


Participants. One hundred five members of an academic research website participated for a chance to win a $\$ 40$ gift certificate.

Procedure. In an online survey, participants rated how confident they were in the direction of adjustment from provided and self-generated anchors. For each of the provided-anchor questions, participants identified the direction of adjustment from the provided anchor (e.g., "Is the average length of a whale greater or less than 69 feet?”), and then they were asked, “How confident are you in your answer?” For each of the self-generated-anchor questions, participants were first asked a question designed to assess whether they knew the correct anchor (e.g., "In which year did the United States declare its independence?”). They were then asked to judge the direction of adjustment (e.g., "Was George Washington elected president before or after the United States declared its independence?”) and to rate their confidence in their answer. Participants rated their confidence on a 7-point scale, with endpoints of $1=$ "not at all confident" and 7 = “absolutely certain.”

The 11 provided-anchor questions and 9 self-generated-anchor questions were presented in separate blocks, with block order counterbalanced across participants. We selected all of the questions and anchors from Epley and Gilovich’s (2001, 2004, 2005, 2006) research comparing self-generated and provided anchors (see Table 4).

\section{Results}

Because all past studies of the difference between self-generated and provided anchors eliminated responses of participants who failed to correctly generate the self-generated anchor (Epley and Gilovich 2001, 2004, 2005, 2006), we did the same. In total, 19.56\% of selfgenerated responses were excluded. ${ }^{5}$ To examine whether participants were more certain in the direction of adjustment from self-generated than from provided anchors, we first averaged 
participants' certainty ratings separately for the provided-anchor and self-generated-anchor items. As predicted, participants were significantly more certain about the direction of adjustment from self-generated anchors $(M=5.60, S E=.11)$ than from provided anchors $(M=$ 4.56, $S E=.12), t(104)=9.73, p<.001$. Across-item analyses on the means displayed in Table 4 similarly show that, on average, the self-generated-anchor items used by previous researchers induced more adjustment-direction certainty than did the provided-anchor items used by those researchers, $t(18)=3.03, p=.007 .^{6}$ Thus, the prior studies that found that motivation is more likely to increase adjustment away from self-generated than provided anchors may have found this because those self-generated anchors confer greater certainty in the direction of adjustment. Study 5: Motivation Increases Adjustment Away From Self-Generated Anchors Only When the

\section{Direction of Adjustment Is Certain}

As noted, the typical finding in the prior literature is that motivation increases adjustment away from self-generated, but not provided, anchors. However, one critical difference between self-generated and provided anchors is that the former typically induce more certainty about the direction of adjustment than do the latter (Study 4). If differences in adjustment-direction certainty explain why self-generated and provided anchors are affected differently by motivation, then motivation should no longer increase adjustment from self-generated anchors if people can be made uncertain (instead of certain) about the direction of adjustment from those anchors. Thus, in Study 5, we manipulated whether a self-generated anchor induced certainty or uncertainty about the direction of adjustment. Following the above reasoning, we predicted that accuracy motivation would increase anchor-estimate gaps only for self-generated anchors that induce certainty in the direction of adjustment.

\section{Method}


Participants. Three hundred thirty-one members of an academic research website participated for a chance to win a \$30 gift certificate.

Procedure. This procedure resembled that of Study 3a. Participants began the online survey by answering a self-generated anchoring question without incentives for accuracy. Those in the certain condition were asked to estimate the highest recorded body temperature in a human being. Those in the uncertain condition were asked to estimate the average body temperature of a bobcat. Unlike in Studies 2 and 3, no anchors were provided to participants; instead, we expected participants in both conditions to generate and use the average body temperature of a human as an anchor when answering these questions. Although participants should know in which direction to adjust from that anchor in the certain condition ("the highest human body temperature is certainly higher than the average human body temperature”), they should be much less sure of the appropriate direction in the uncertain condition ("are bobcats warmer or cooler than the average human?”).

After answering this question, participants moved on to a new screen that informed them that they could revise their prior estimates and that an accurate estimate would earn them entry into a drawing for a \$100 amazon.com gift card. They were then given a chance to revise their estimates. For example, a participant who originally estimated the highest recorded human body temperature to be 105 degrees Fahrenheit read:

You indicated that the highest recorded body temperature in a human being was 105 degrees Fahrenheit. You now have a chance to revise this estimate in order to make it more accurate.

If you accurately answer this question, you will earn a chance to win a $\$ 100$ amazon gift card! If you do not wish to revise your estimate, please just type the answer that you gave previously. What is the highest recorded body temperature in a human being? 
After generating their revised estimates, participants were asked to indicate the average body temperature of a healthy human being (to assess whether they knew the anchor), and they were asked whether they had thought of this number when generating the earlier estimate (Epley \& Gilovich, 2005). They then indicated the direction of adjustment from the self-generated anchor (e.g., "Is the average temperature of a healthy human being greater or less than the average body temperature of a bobcat?”), and they rated how certain they were in their answer to that question (1 = completely uncertain; 9 = completely certain). Finally, we asked participants to indicate whether they looked up the answer while completing the survey. We assured them that their earnings would be unaffected by how they answered this final question.

\section{Results}

We eliminated $6.0 \%$ of participants because they indicated looking up the answer while completing the survey. Following Epley and Gilovich (2001, 2004, 2005, 2006), we also excluded $11.1 \%$ of participants for failing to generate the correct anchor (i.e., people who thought that the average human body temperature was something other than 98-99 degrees Fahrenheit), and another $11.5 \%$ of participants for indicating that they did not use the anchor. None of these percentages differed by condition, meaning, for example, that participants who estimated the bobcat's average body temperature were just as likely as those who estimated the highest recorded human body temperature to correctly generate and report using the average human body temperature as an anchor. We were left with 225 participants for the final analysis, 110 in the certain condition and 115 in the uncertain condition.

We successfully manipulated whether participants were certain about the direction of adjustment: Participants in the certain condition were more certain about the direction of 
adjustment $(M=8.35, S E=.17)$ than were participants in the uncertain condition $(M=3.90, S E$ $=.21), \mathrm{t}(223)=16.17, p<.001$.

To analyze the effect of motivation on anchor-estimate gaps, we first computed the difference between participants’ unmotivated initial estimates and their incentive-motivated revised estimates, and then we converted these differences into standard deviation units (by dividing each difference by the standard deviation of the item's difference scores). This resulted in a motivation score that reflected how far newly-motivated participants moved away from (or back toward) the anchor. As in Study 3a, this score was positive if motivation increased the anchor-estimate gap, negative if motivation decreased the anchor-estimate gap, and zero if the two estimates were identical.

A t-test on these motivation scores revealed the predicted effect, $t(223)=3.42, p<.001$ : Motivation increased anchor-estimate gaps more in the certain $(M=0.24, S E=.10)$ than in the uncertain condition $(M=-0.21, S E=.09)$. Replicating Epley and Gilovich (2005), motivation increased the anchor-estimate gap when people estimated the highest recorded temperature in a human being (and were thus certain about the direction of adjustment from the self-generated anchor), $t(109)=2.52, p<.02$. However, when people estimated the average temperature of a bobcat (and were thus uncertain about the direction of adjustment from that same self-generated anchor), motivation significantly decreased the anchor-estimate gap, $t(114)=-2.31, p<.03 .^{7}$

Thus, whereas Studies 1 through 3 showed that provided anchors can "behave” like selfgenerated anchors when participants are certain about the direction of adjustment, Study 5 shows that self-generated anchors can "behave” like provided anchors when participants are uncertain about the direction of adjustment. These findings suggest that certainty about the direction of adjustment is an important difference between self-generated and provided anchors, a difference 
that can perhaps explain why self-generated anchors are typically affected by increasing motivation, but why provided anchors typically are not. Indeed, our results suggest that adjustment is an important process underlying responding to both types of anchors, and that the anchors may not, as previously assumed, give rise to fundamentally different types of processing.

\section{General Discussion}

In this paper, we have revealed how accuracy motivation affects anchoring and adjustment. More specifically, we have shown that people are generally more certain about the direction of adjustment from self-generated than from provided anchors (Study 4), and that, when people are certain (vs. uncertain) about the direction of adjustment from an anchor, they are more likely to conclude that they have insufficiently adjusted from that anchor (Study 1). Most important, we have shown that certainty about the direction of adjustment - and not anchor type - determines whether accuracy motivation will increase adjustments from an anchor: Motivated participants adjust further away from anchors as long as they are certain about the direction of adjustment, regardless of whether the anchor is provided or self-generated (Studies 2, 3, and 5). These results have important theoretical implications. In what follows, we discuss what these findings suggest about current theories of anchoring. Anchoring and Adjustment Theory

According to anchoring-and-adjustment theory, people effortfully adjust away from anchor values, and motivation increases the tendency to adjust more than usual - that is, to carefully revise estimates. According to existing versions of this theory, adjustment involves moving further from anchor values; hence, motivation should increase the gap between anchors and final estimates and decrease anchoring effects. However, this prediction has not often been supported, as accuracy motivation has rarely been shown to reduce anchoring effects (Chapman \& Johnson, 
2002). This discrepancy between the theory's predictions and empirical findings caused many researchers to abandon anchoring and adjustment as an explanation of how provided anchors affect judgment. Indeed, many researchers believe that people do not adjust from provided anchors (e.g., Chapman \& Johnson, 2002; Epley \& Gilovich, 2006; Mussweiler \& Strack, 1999).

In contrast, we have proposed that people do adjust from all anchor values, but that one must reconsider how motivation affects adjustment. We suggested that the process of adjustment can proceed in multiple directions. For example, after initially adjusting upward from a low anchor value, a respondent can conclude that her initial adjustment was too extreme, and thereby adjust her estimate by retreating back toward the anchor. If one accepts this (admittedly simple) notion, then motivation should increase the gap between anchors and final estimates only when participants believe they have insufficiently adjusted from the anchor values in the first place. If this revised view of adjustment is accurate, then the effect of accuracy motivation on the size of the anchor-estimate gap should depend heavily on people's beliefs about the sufficiency of their initial adjustments.

To investigate this notion, we identified one factor that influences these beliefs: certainty about the direction of adjustment. We predicted that being uncertain (vs. certain) about the direction of adjustment will more often spur the belief that one has adjusted too far or perhaps in the wrong direction. Consistent with this prediction, Studies 1a and 1b showed that people who knew the correct direction of adjustment were more likely to believe they insufficiently adjusted from anchor values than people for whom the correct direction of adjustment was much less obvious. Further, consistent with Studies 1a and $1 \mathrm{~b}$ and with our revised view of anchoring and adjustment, Studies 2, 3, and 5 showed that accuracy motivation moved estimates further from anchors, but that it did so only when people were certain about the direction of adjustment. When 
people were uncertain about the direction of adjustment, accuracy motivation either exerted no consistent effect (Study 2), or brought estimates significantly nearer to anchors (Studies 3 and 5). Thus, prior studies that found no effect of motivation on provided anchors (and that therefore concluded that adjustment does not operate for provided anchors) may have been hindered by participants' uncertainty about the direction in which to adjust from the anchors. ${ }^{8}$ We show that motivation can, under specific theoretically-derived conditions, produce measurable effects on responses to provided anchors. In so doing, we provide strong support for the notion that people do effortfully adjust from provided anchor values.

\section{Self-Generated vs. Provided Anchors}

Previous research has emphasized a distinction between self-generated anchors and those that are externally provided (Epley \& Gilovich, 2001, 2004, 2005, 2006). Anchoring-and-adjustment theory is believed to explain how self-generated anchors affect judgment, whereas the selective accessibility model is believed to explain the effects of provided anchors. As noted, this distinction was based largely on the fact that motivation did little to moderate the effects of provided anchors, but had reliable effects when anchors were self-generated. Our findings suggest, however, that such disparate effects of motivation arose not because self-generated and provided anchors induce fundamentally different judgmental processes, but rather because people are typically uncertain about which way to adjust from provided anchors but certain about which way to adjust from self-generated anchors (Study 4). Indeed, no matter whether the anchors were provided or self-generated, motivation increased anchor-estimate gaps when participants were certain about in which direction to adjust (Studies 2, 3a, 3b, and 5). On the basis of these findings, we believe that the distinction between self-generated and provided anchors is unnecessary for understanding the types of processing underlying anchoring effects. 
(Of course, the distinction between these anchor types remains interesting for other reasons, such as for understanding when anchoring might arise). A more parsimonious theory is likely appropriate - a theory that assumes that all anchor types induce the same psychological processes, processes that we believe are captured both by anchoring-and-adjustment and selective accessibility.

We should note that the distinction between self-generated and externally-provided anchors has been supported by studies investigating variables other than accuracy motivation. Indeed, research has more generally shown that variables that increase thinking (e.g., horizontal head movement, need for cognition) increase adjustment away from self-generated anchors but not from provided anchors, whereas variables that decrease thinking (e.g., vertical head movement, alcohol use) decrease adjustment away from self-generated anchors but not from provided anchors (Epley \& Gilovich, 2001, 2004, 2006). These findings are completely consistent with our framework: Although this paper focused on the effects of accuracy motivation on anchoring and adjustment, we, like all anchoring-and adjustment theorists, predict that any variable that increases thinking will increase people’s tendency to revise their initial estimates. Moreover, we predict that such variables will prompt people to revise their estimates further from the anchor only when they are certain about the direction of adjustment (or when they believe, for any other reason, that their initial adjustments are insufficient). Because it is very likely that only selfgenerated anchors conferred participants with certainty about the direction of adjustment in prior studies (see Study 4), our theory is consistent with the fact that variables that increased thinking (e.g., horizontal head movement) increased adjustment away from self-generated anchors only. However, we also predict that similar effects will emerge for provided anchors whenever people are certain about which direction to adjust from such anchors. In sum, we believe that our theory 
can explain all of these prior discrepancies between provided and self-generated anchors, while doing so parsimoniously, without assuming that different anchors induce different processes. ${ }^{9}$ Selective Accessibility Model

The selective accessibility model (Mussweiler \& Strack, 1999, 2000; Strack \& Mussweiler, 1997) is widely considered to be the best explanation of how provided anchors affect judgment. Indeed, there is much evidence supporting the model's signature claim, which is that the act of considering anchor values increases the accessibility of anchor-consistent information, which in turn changes which values people believe to be plausible (see Epley 2004, for a review). We do not dispute any of this evidence. We feel, however, that our data strongly suggest that the processes outlined by the selective accessibility model are not the only processes that underlie the effects of provided anchors. After all, ex ante, the selective accessibility model does not predict that motivation will affect the size of anchor-estimate gaps (see Chapman \& Johnson, 2002). In fact, proponents of the selective accessibility model have often cited the null effect of motivation on anchoring as a finding that is consistent with their theory and have never suggested that motivation should affect anchor-estimate gaps (e.g., Chapman \& Johnson, 2002; Mussweiler \& Englich, 2005; Mussweiler \& Strack, 1999).

Ultimately, the selective accessibility model is silent about which variables affect the size of anchor-estimate gaps and about the process by which initial estimates are revised. Because of this, the selective accessibility model cannot predict the results reported in this paper, but neither is it undermined by these results.

\section{Toward an Integrative Theory of Anchoring}

As stated in the introduction, whereas many theorists assume that the selective accessibility model and anchoring-and-adjustment theory constitute competing explanations of anchoring, 
they are not logically contradictory, and we think it is more appropriate to consider them as complementary accounts that both contribute to anchoring effects. Indeed, on the one hand, the selective accessibility model (but not anchoring-and-adjustment theory) can nicely account for a finding that is often replicated - namely, merely considering an anchor increases the perceived plausibility of values that are close to the anchor. ${ }^{10}$ On the other hand, our revision of anchoringand-adjustment theory (but not the selective accessibility model) can nicely account for the fact that motivation can, under predictable conditions, increase anchor-estimate gaps and decrease anchoring effects (as shown here). Neither theory can explain both of these empirical regularities (but neither of them is threatened by them, either). Thus, we believe that both theories are useful and necessary, and that they must co-exist to provide a more complete account of anchoring effects (see Figure 2b).

We further suggest that selective accessibility and effortful adjustment processes operate independently when anchors are encountered. Merely considering an anchor may lead people to selectively recruit anchor-consistent information, and reliance on that information may increase the perceived plausibility of estimates close to the anchor. At the same time, people settle on final estimates by effortfully adjusting away from (and possibly back toward) anchors. ${ }^{11}$ This integrative theory is more parsimonious than existing theories because it does not assume that different anchors (self-generated vs. experimenter-provided; plausible vs. implausible) exert fundamentally different effects on judgment. Moreover, it is the only theory that can parsimoniously account for existing evidence and the evidence presented in this article.

This integrative theory suggests a refreshingly clear and new agenda for future anchoring research. Rather than debating which of anchoring's many theories are correct, researchers should look for ways to integrate and refine them. For example, questions about whether 
adjustment happens should give way to questions about how adjustment operates (e.g., Janiszewski \& Uy, 2008). We look forward to future research that proceeds in this spirit. 


\section{References}

Carlson, B. W. (1990). Anchoring and adjustment in judgments under risk. Journal of Experimental Psychology: Learning, Memory, and Cognition, 16, 665-676.

Chapman, G. B., \& Bornstein, B. H. (1996). The more you ask for, the more you get: Anchoring in personal injury verdicts. Applied Cognitive Psychology, 10, 519-540.

Chapman, G. B., \& Johnson, E. J. (2002). Incorporating the irrelevant: Anchors in judgment of belief and value. In T. Gilovich, D. Griffin, \& D. Kahneman (Eds.), Heuristics and biases: The psychology of intuitive judgment (pp. 120-138). Cambridge, England: Cambridge University Press.

Englich, B., Mussweiler, T., \& Strack, F. (2006). Playing dice with criminal sentences: The influence of irrelevant anchors on experts’ judicial decision making. Personality and Social Psychology Bulletin, 32, 188-200.

Epley, N. (2004). A tale of tuned decks? Anchoring as accessibility and anchoring as adjustment. In D. J. Koehler \& N. Harvey (Eds.), Blackwell handbook of judgment and decision making (pp. 240-256). Malden, MA: Blackwell Publishing.

Epley, N., \& Gilovich, T. (2001). Putting adjustment back in the anchoring and adjustment heuristic: Differential processing of self-generated and provided anchors. Psychological Science, 12, 391-396.

Epley, N., \& Gilovich, T. (2004). Are adjustments insufficient? Personality and Social Psychology Bulletin, 30, 447-460.

Epley, N., \& Gilovich, T. (2005). When effortful thinking influences judgmental anchoring: Differential effects of forewarning and incentives on self-generated and externally-provided anchors. Journal of Behavioral Decision Making, 18, 199-212. 
Epley, N., \& Gilovich, T. (2006). The anchoring-and-adjustment heuristic: Why the adjustments are insufficient. Psychological Science, 17, 311-318.

Epley, N., Keysar, B., Van Boven, L., \& Gilovich, T. (2004). Perspective taking as egocentric anchoring and adjustment. Journal of Personality and Social Psychology, 87, 327-339.

Galinsky, A. D., \& Mussweiler, T. (2001). First offers as anchors: The role of perspective-taking and negotiator focus. Journal of Personality and Social Psychology, 81, 657-669.

Gilbert, D. T. (2002). Inferential correction. In T. Gilovich, D. Griffin, \& D. Kahneman (Eds.), Heuristics and biases: The psychology of intuitive judgment (pp. 167-184). Cambridge, England: Cambridge University Press.

Green, D., Jacowitz, K. E., Kahneman, D., \& McFadden, D. (1998). Referendum contingent valuation, anchoring, and willingness to pay for public goods. Resource and Energy Economics, 20, 85-116.

Grice, H. P. (1975). Logic and conversation. In P. Cole \& J. L. Morgan (Eds.), Syntax and semantics: Speech acts (pp. 41-58). New York: Academic Press.

Jacowitz, K. E., \& Kahneman, D. (1995). Measures of anchoring in estimation tasks. Personality and Social Psychological Bulletin, 21, 1161-1167.

Janiszewski, C. \& Uy, D. (2008). Precision of the anchor influences the amount of adjustment. Psychological Science, 19, 121-127.

Klayman, J., \& Ha, Y. W. (1987). Confirmation, disconfirmation, and information in hypotheses testing. Psychological Review, 94, 211-228.

Mussweiler, T., \& Englich, B. (2005). Subliminal anchoring: Judgmental consequences and underlying mechanisms. Organizational Behavior and Human Decision Processes, 98, 133143. 
Mussweiler, T., \& Strack F. (1999). Hypothesis-consistent testing and semantic priming in the anchoring paradigm: A selective accessibility model. Journal of Experimental Social Psychology, 35, 136-164.

Mussweiler, T., \& Strack, F. (2000). The use of category and exemplar knowledge in the solution of anchoring tasks. Journal of Personality and Social Psychology, 78, 1038-1052.

Northcraft, G. B., \& Neale, M. A. (1987). Experts, amateurs, and real estate: An anchoring-andadjustment perspective on property pricing decisions. Organizational Behavior and Human Decision Processes, 39, 84-97.

Quattrone, G. A. (1982). Overattribution and unit formation: When behavior engulfs the person. Journal of Personality and Social Psychology, 42, 593-607.

Schwarz, N. (1996). Cognition and communication: Judgmental biases, research methods, and the logic of conversation. Mahwah, NJ: Lawrence Erlbaum Associates.

Simmons, J. P., \& Nelson, L. D. (2006). Intuitive confidence: Choosing between intuitive and nonintuitive alternatives. Journal of Experimental Psychology: General, 135, 409-428.

Simonson, I., \& Drolet, A. (2004). Anchoring effects on consumers’ willingness-to-pay and willingness-to-accept. Journal of Consumer Research, 31, 681-690.

Stewart, N. (2009). The cost of anchoring on credit-card minimum repayments. Psychological Science, 20, 39-41.

Strack, F., \& Mussweiler, T. (1997). Explaining the enigmatic anchoring effect: Mechanisms of selective accessibility. Journal of Personality and Social Psychology, 73, 437-446.

Tversky, A., \& Kahneman, D. (1974). Judgment under uncertainty: Heuristics and biases. Science, 185, 1124-1131. 
Wansink, B., Kent, R. J., \& Hoch, S. J. (1998). An anchoring and adjustment model of purchase quantity decisions. Journal of Marketing Research, 35, 71-81.

Wason, P. C. (1960). On the failure to eliminate hypotheses in a conceptual task. Quarterly Journal of Experimental Psychology, 12, 129-140.

Wegener, D. T., \& Petty, R. E. (1995). Flexible correction processes in social judgment: The role of naïve theories in corrections for perceived bias. Journal of Personality and Social Psychology, 68, 36-51.

Wright, W. F., \& Anderson, U. (1989). Effects of situation familiarity and financial incentives on use of the anchoring and adjustment heuristic for probability assessment. Organizational Behavior and Human Decision Processes, 44, 68-82. 


\section{Footnotes}

1. The results of Study 1a are not entirely in line with our predictions. Because fewer than half of those who knew the direction of adjustment believed they had adjusted insufficiently, these data cannot explain why increasing accuracy motivation typically increases adjustment away from self-generated anchors, for which the direction of adjustment is typically known (Epley \& Gilovich, 2005). One possible explanation is that because participants in Study 1a were not told that the anchors were uninformative, some believed that the correct answers were very close to the anchors, and thus that they had over-adjusted. We remedied this in Study $1 \mathrm{~b}$ by telling participants that the anchors were uninformative. Collapsing across both items in Study 1b, participants who were certain about the direction of adjustment were more likely to believe that they had adjusted insufficiently than that they had overadjusted, $\chi^{2}(1, N$ $=195)=4.31, p<.03$.

2. For 6 of the 8 questions, Stage 1 estimates from implausible anchors were significantly further from accuracy than estimates from plausible anchors (i.e., there was a bigger anchoring effect for the implausible anchors; see Strack and Mussweiler, 1997, for the same effect). However, this effect did not account for the differential effect of motivation on anchor-estimate gaps for plausible versus implausible anchors. By-item analyses on adjusted means that co-varied out Stage 1 estimates yielded the same results reported earlier: Incentives increased anchor-estimate gaps more for implausible than plausible anchors, $t(7)=$ 5.73, $p=.001$. This rules out the possibility that incentives increased anchor-estimate gaps for implausible anchors mainly because those anchors were more likely to elicit initially insufficient adjustments. 
3. The first 18 participants received a questionnaire that contained an error on the sixth and final question (about New York City's temperature), and the error rendered any responses to this question meaningless. Thus, we analyzed these participants’ responses to only the first five questions.

4. These results were consistent across items. Accuracy motivation directionally increased anchor-estimate gaps for five out of six items when the anchors were implausible, but directionally decreased anchor-estimate gaps for five out of six items when the anchors were plausible. Moreover, despite the use of a small number of items in this study, a by-item Plausibility x Motivation repeated-measures ANOVA revealed the predicted interaction, $F(1$, 5) $=7.99, p<.04$.

5. This exclusion rate is very similar to other self-generated anchoring studies. For example, Epley and Gilovich (2001, Study 2) excluded 20.67\% of their participants using the same criteria.

6. Table 4 reveals one oddity. Although, logically, it must be true that the second explorer after Columbus landed in the West Indies after Columbus did, participants were not very confident about this. We speculate that this lack of confidence arose because the question's surprising obviousness ("Did the second European explorer, after Columbus, land in the West Indies before or after Columbus first landed in the West Indies?”) led some participants to interpret this question differently than we intended or to worry that this was a “trick question.” A clearer wording may have yielded more certainty about the direction of adjustment.

7. Additional analyses showed that certainty in the direction of the adjustment correlated positively with participants’ motivation scores, $r(223)=.15, p<.03$. 
8. Although prior studies have typically found a null effect of motivation on provided anchors, strictly speaking, our theory predicts that motivation will decrease anchor-estimate gaps when participants are uncertain about the direction of adjustment. However, if participants are differentially certain about the direction of adjustment (i.e., if some are certain and some are uncertain) or if the items in a study differentially generate certainty about the direction of adjustment (i.e., if some generate certainty and some generate uncertainty), then motivation may exert no consistent effect on adjustment, resulting in the observed null effects. It is also worth noting that null effects of motivation may arise because of measurement error; indeed, many anchoring questions generate highly variable answers, and this variance strongly decreases the probability of detecting an effect of motivation on adjustment.

9. Epley and Gilovich (2006) have recently found that people's estimates reside in the anchor half of the plausible range of target values for self-generated anchors but not for provided anchors (Epley \& Gilovich, 2006). This is the newest finding in the self-generated vs. provided anchor literature, and it is believed to constitute evidence for the operation of effortful adjustment: That is, for any estimate, people believe that a range of values is plausible. According to Epley and Gilovich, anchoring-and-adjustment theory predicts that, if people are truly adjusting from an anchor, they will terminate their adjustments somewhere in the half of the plausible range that is closest to the anchor value. Epley and Gilovich (2006) report that estimates reside in the anchor half of the plausible range of values only when anchors are self-generated, but we have recently conducted a study that shows that estimates from provided anchors can also reside in the anchor half of the plausible range, especially when participants are certain about the direction of adjustment. This again suggests that differences in adjustment-direction certainty may underlie the observed 
differences between self-generated and provided anchors. Please contact the first author for a complete summary of this study.

10. Revisions to the selective accessibility model have been proposed in order to explain the oftreplicated finding that anchoring effects tend to be larger when anchors are extreme than when they are moderate (e.g., Mussweiler \& Strack, 1999). However, if, as our reading of the selective accessibility model suggests, the mere act of considering an anchor increases the plausibility of values that are close to the anchor, then considering an extreme (moderate) anchor will make extreme (moderate) values seem more plausible, thereby making extreme (moderate) estimates more likely. And, of course, more extreme estimates translate into bigger anchoring effects.

11. Although we think it is safest to assume that selective accessibility and effortful adjustment processes operate independently, it is interesting to consider ways in which they might interact. One speculation is that the consideration of accessible anchor-consistent information may increase the perceived plausibility of values that are close to the anchor, which may, when the direction of adjustment is not specified, increase uncertainty in the direction of adjustment. This may, in turn, decrease the propensity to believe that one's initial adjustment is insufficient, and therefore decrease the tendency for accuracy motivation to elicit adjustments that are even further away from the anchor. Thus, it is possible that selective accessibility processes influence adjustment processes by increasing adjustment-direction uncertainty. 
Table 1

Study 2: Questions and Anchors

\begin{tabular}{cccc}
\hline Question & Low Anchor & High Anchor & Correct Answer \\
\hline Length of Mississippi River (miles) & 1,200 & 3,500 & 2,320 \\
Average annual rainfall in Philadelphia (inches) & 25 & 65 & 41 \\
Year James K. Polk began his term as U.S. President & 1815 & 1875 & 1845 \\
Maximum speed of a house cat (miles per hour) & 5 & 55 & 30 \\
Average annual temperature in Phoenix (degrees Fahrenheit) & 52 & 94 & 73 \\
Population of Chicago & 800,000 & $5,000,000$ & $2,900,000$ \\
Height of Mount Everest (feet) & 13,000 & 45,000 & 29,032 \\
Average lifespan of a bullfrog (years) & 2 & 30 & 16 \\
Number of countries in the world & 55 & 330 & 192 \\
Distance between San Francisco and Kansas City (miles) & 600 & 3,000 & 1800 \\
\hline
\end{tabular}


Table 2

Study 3a: Questions and Anchors

\begin{tabular}{|c|c|c|c|}
\hline Question & Plausible Anchor & Implausible Anchor & Correct Answer \\
\hline Year Seinfeld first aired & 1995 & 2005 & 1989 \\
\hline Average temperature in Montreal in December & $39^{\circ} \mathrm{F}$ & $85^{\circ} \mathrm{F}$ & $26^{\circ} \mathrm{F}$ \\
\hline Jack Nicholson's birth year & 1945 & 1977 & 1937 \\
\hline Average temperature in Phoenix in August & $90^{\circ} \mathrm{F}$ & $50^{\circ} \mathrm{F}$ & $105^{\circ} \mathrm{F}$ \\
\hline Year The Godfather appeared in theaters & 1978 & 1991 & 1972 \\
\hline Average temperature in Los Angeles in July & $76^{\circ} \mathrm{F}$ & $43^{\circ} \mathrm{F}$ & $84^{\circ} \mathrm{F}$ \\
\hline Jennifer Lopez's birth year & 1975 & 1988 & 1970 \\
\hline Average temperature in Boston in January & $42^{\circ} \mathrm{F}$ & $86^{\circ} \mathrm{F}$ & $36^{\circ} \mathrm{F}$ \\
\hline
\end{tabular}


Table 3

Study 3b: Questions and Anchors

\begin{tabular}{cccc}
\hline Question & Plausible Anchor & Implausible Anchor & Correct Answer \\
\hline Year Seinfeld first aired & 1993 & 2007 & 1989 \\
Average temperature in Boston in January & $39^{\circ} \mathrm{F}$ & $90^{\circ} \mathrm{F}$ & $36^{\circ} \mathrm{F}$ \\
Year JFK began his term as U.S. President & 1965 & 1995 & 1961 \\
Average temperature in Phoenix in August & $96^{\circ} \mathrm{F}$ & $20^{\circ} \mathrm{F}$ & $105^{\circ} \mathrm{F}$ \\
Year Back To The Future appeared in theaters & 1982 & 1940 & 1985 \\
Average temperature in New York in September & $70^{\circ} \mathrm{F}$ & $18^{\circ} \mathrm{F}$ & $74^{\circ} \mathrm{F}$ \\
\hline
\end{tabular}


Table 4

Study 4: Questions, Anchors, and Results

\begin{tabular}{|c|c|c|}
\hline Item & Anchor & $\begin{array}{c}\text { Adjustment-Direction } \\
\text { Certainty }\end{array}$ \\
\hline \multicolumn{3}{|c|}{$\underline{\text { Self-Generated }}$} \\
\hline Year of $2^{\text {nd }}$ explorer after Columbus & 1492 & 4.45 \\
\hline George Washington's election year & 1776 & 5.20 \\
\hline Boiling point of water on Mt. Everest & $212^{\circ} \mathrm{F}$ & 5.24 \\
\hline Days for Mars to orbit sun & 365 & 5.34 \\
\hline Gestation period of an elephant & 9 months & 5.49 \\
\hline Freezing point of vodka & $32^{\circ} \mathrm{F}$ & 5.71 \\
\hline Lowest recorded human temp. & $98.6^{\circ} \mathrm{F}$ & 6.32 \\
\hline Highest recorded human temp. & $98.6^{\circ} \mathrm{F}$ & 6.43 \\
\hline Number of U.S. states in 1840 & 50 & 6.48 \\
\hline Average & & 5.6 \\
\hline \multicolumn{3}{|c|}{$\underline{\text { Provided }}$} \\
\hline Number of female profs at Berkeley & 130 & 3.29 \\
\hline Number of nations in UN & 127 & 3.77 \\
\hline Length of Mississippi River & 2,000 miles & 3.88 \\
\hline Average length of a whale & 69 feet & 4.12 \\
\hline Maximum speed of a housecat & $7 \mathrm{mph}$ & 4.19 \\
\hline Height of Mt. Everest & 45,500 feet & 4.54 \\
\hline Average winter temp. in Antarctica & $1^{\circ} \mathrm{F}$ & 4.61 \\
\hline Year telephone invented & 1920 & 4.74 \\
\hline Height of tallest redwood & 65 feet & 5.02 \\
\hline Number of U.S. babies born per day & 100 & 5.89 \\
\hline Population of Chicago & 200,000 & 6.11 \\
\hline Average & & 4.6 \\
\hline
\end{tabular}

Note. Adjustment-direction certainty was rated on a 7-point scale $(1=$ not at all confident and $7=$ absolutely certain). 


\section{Figure Captions}

1. Anchoring-and-Adjustment Theory: Current and Revised

2. The Theoretical Relationship Between Selective Accessibility and Anchoring and Adjustment: Current and Revised

3. Studies 1a and 1b: Percentage of Participants Believing Their Initial Adjustments Were Insufficient

4. Study 2: The Effect of Motivation on Anchor-Estimate Gaps

5. Study 2: The Effect of Motivation on Anchoring

6. Study 3a: The Effect of Motivation on Anchor-Estimate Gaps

7. Study 3b: The Effect of Motivation on Anchor-Estimate Gaps 
Figure la

\section{Anchoring-and-Adjustment}

(Current)

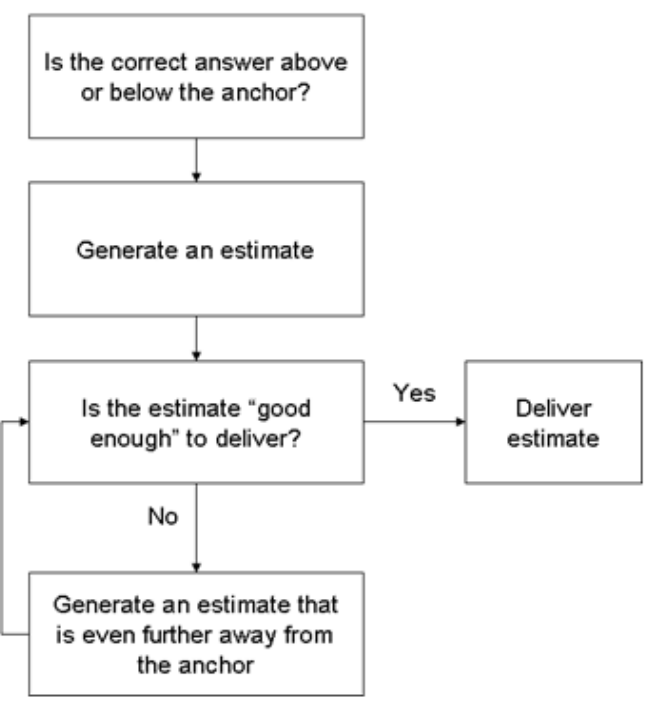

Figure 1b

Anchoring-and-Adjustment (Revised)

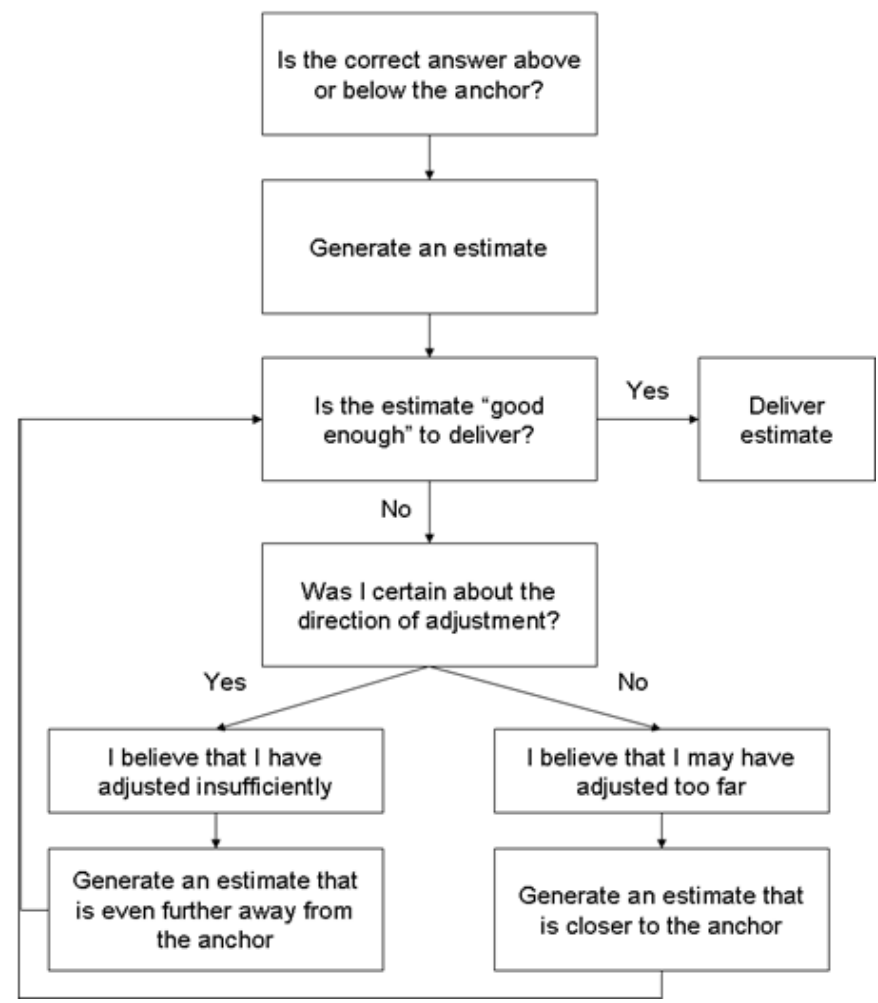

Note. Figure 1b is not meant to imply that adjustment-direction certainty is the only determinant of beliefs about the sufficiency of adjustment. There are likely many such determinants. 
Do People Adjust From Provided Anchors? 52

Figure 2a

Current: Different Theories For Different Anchors

Anchor is Provided

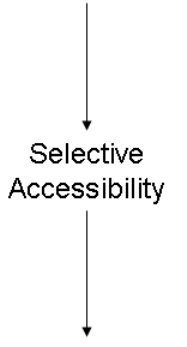

Final Estimate
Anchor is Self-Generated

Anchoring and

Adjustment

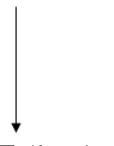

Figure $2 \mathrm{~b}$

Revised: Integrative Theory of Anchoring

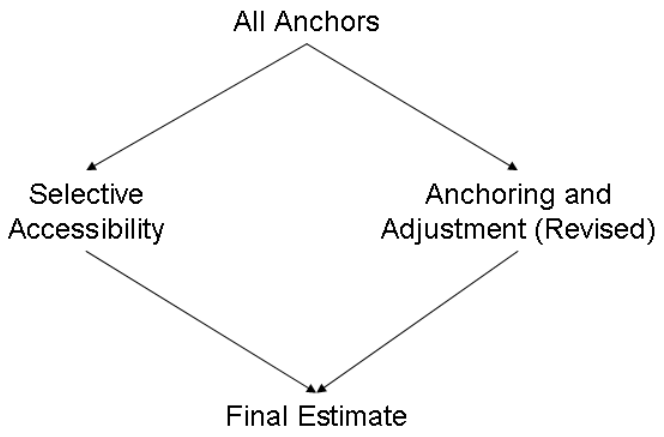



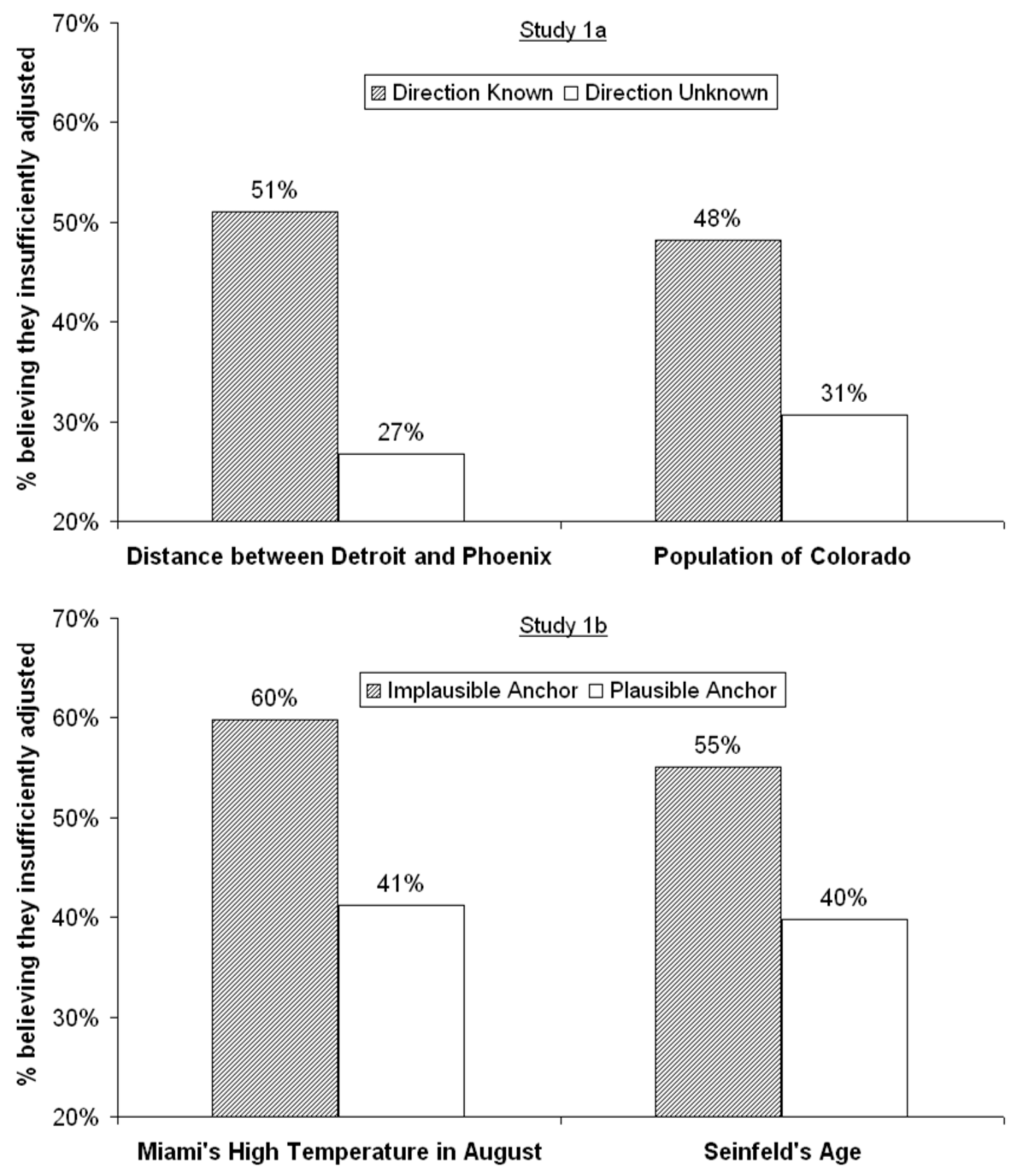


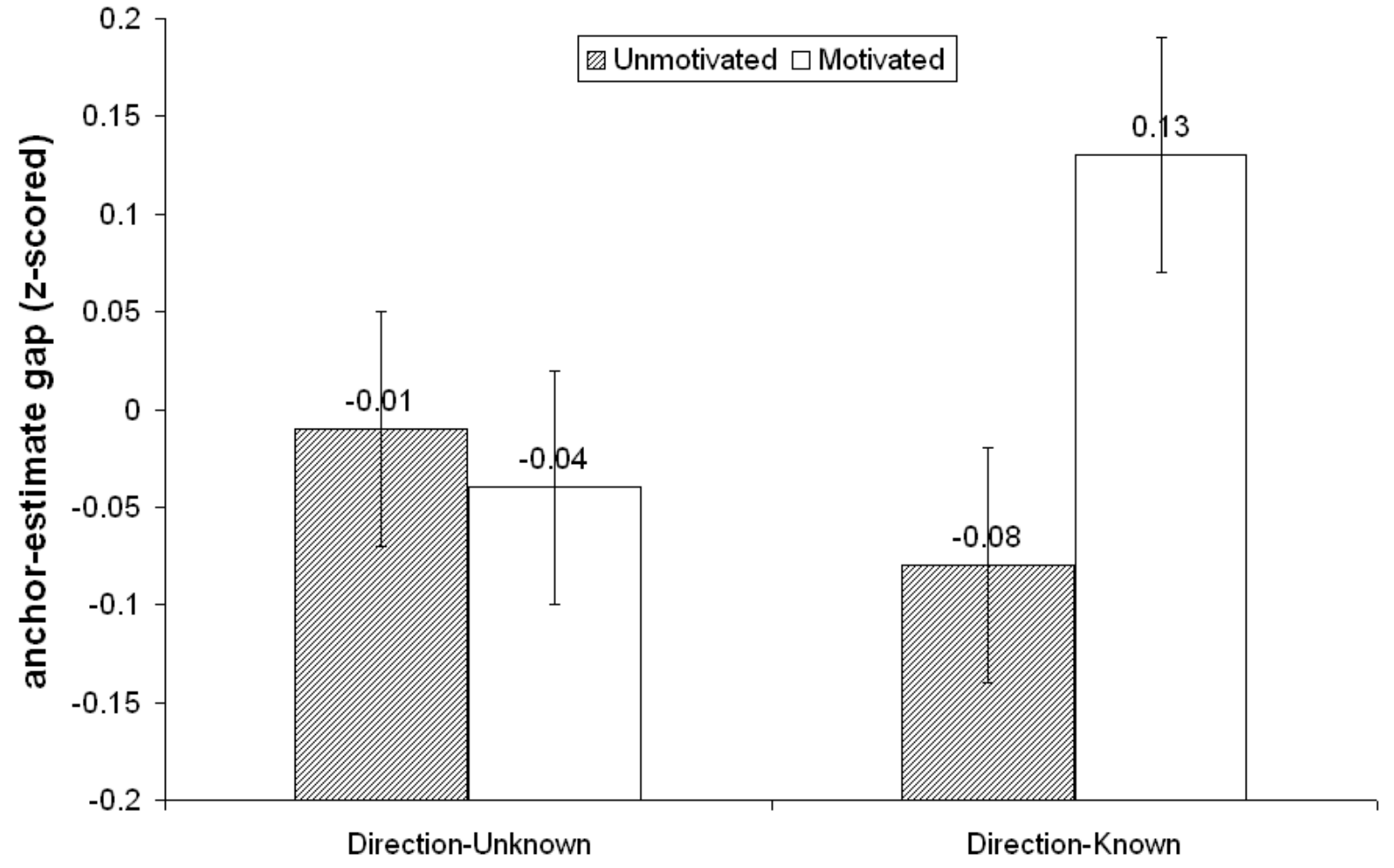



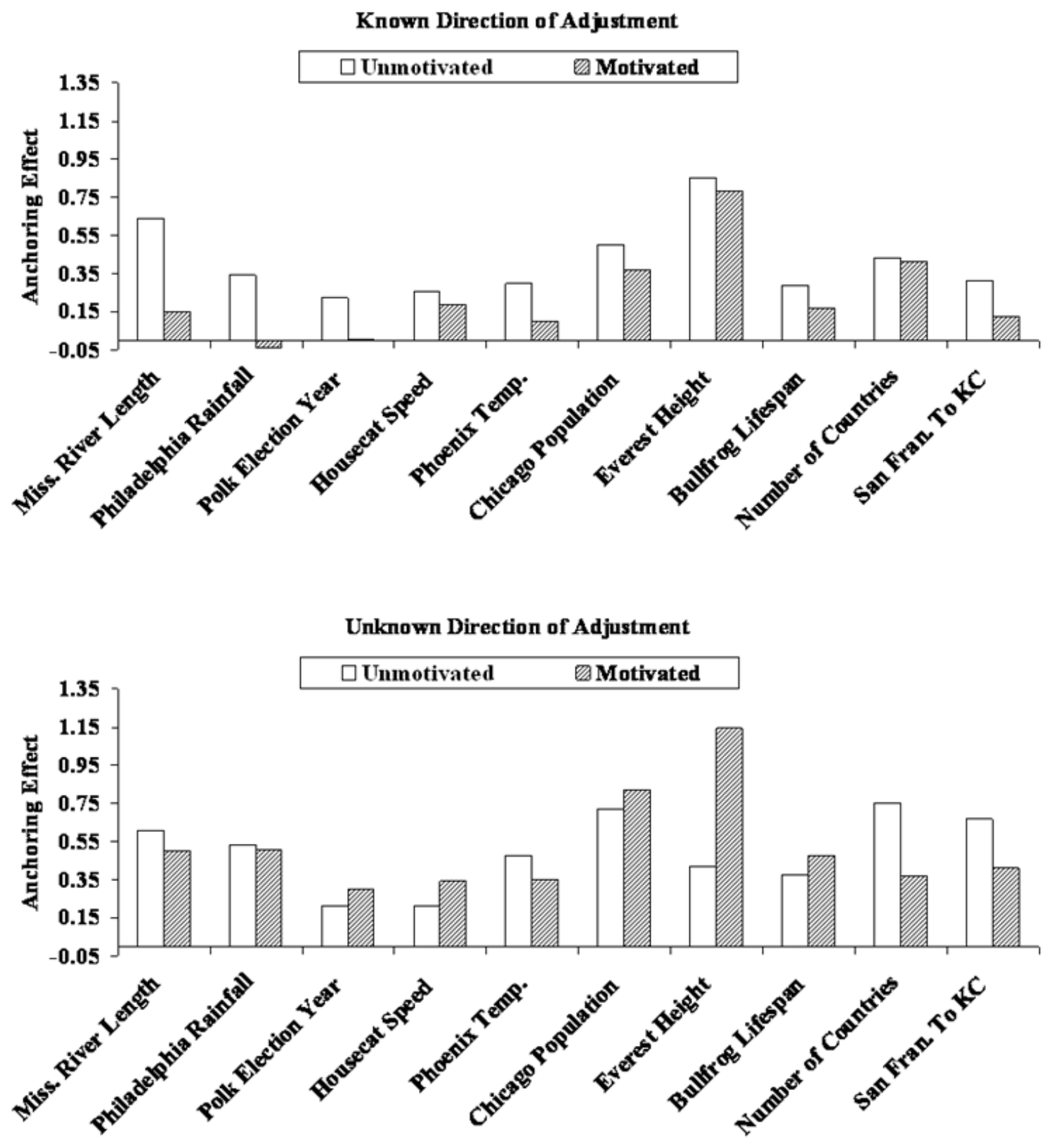


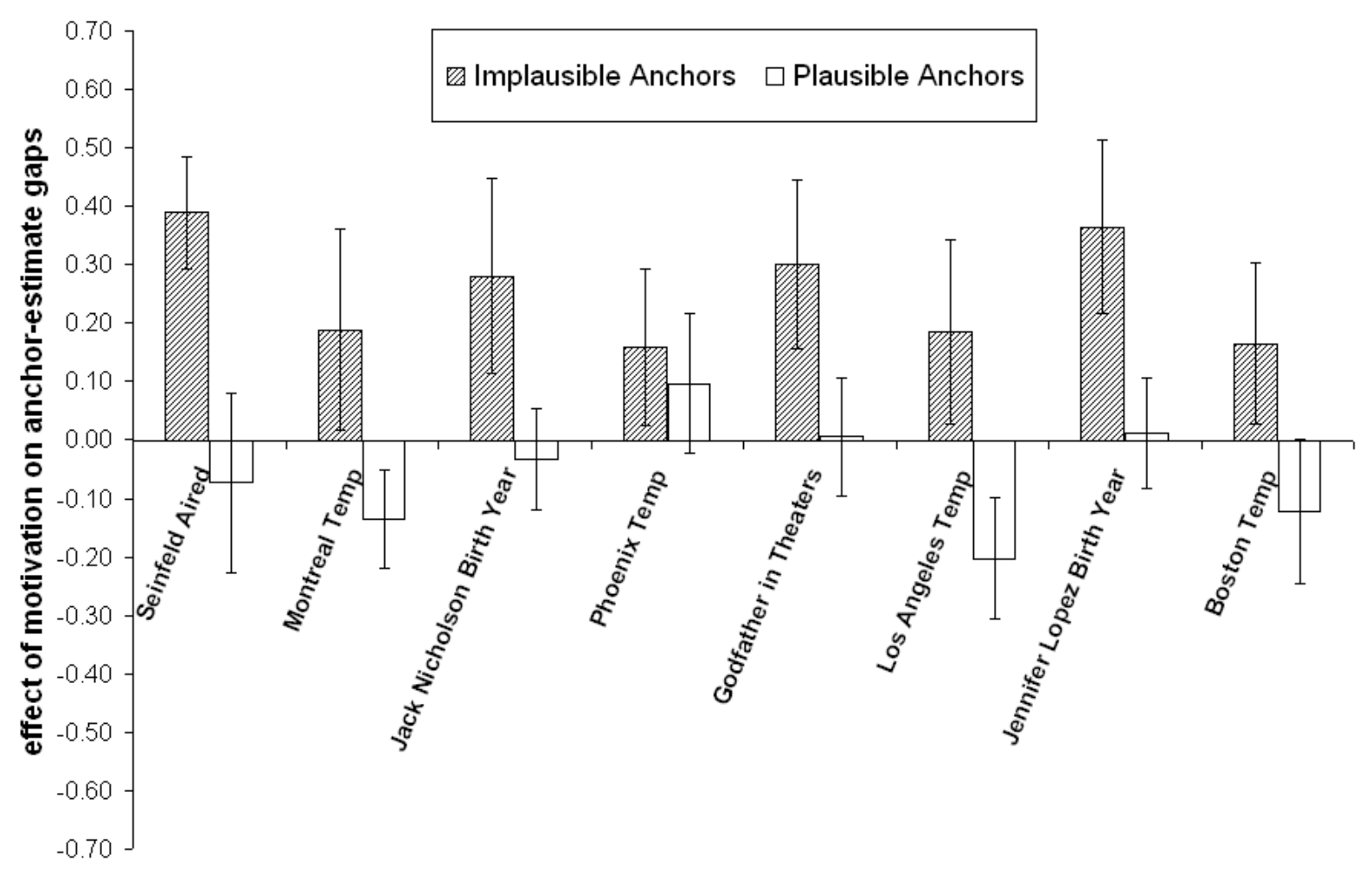




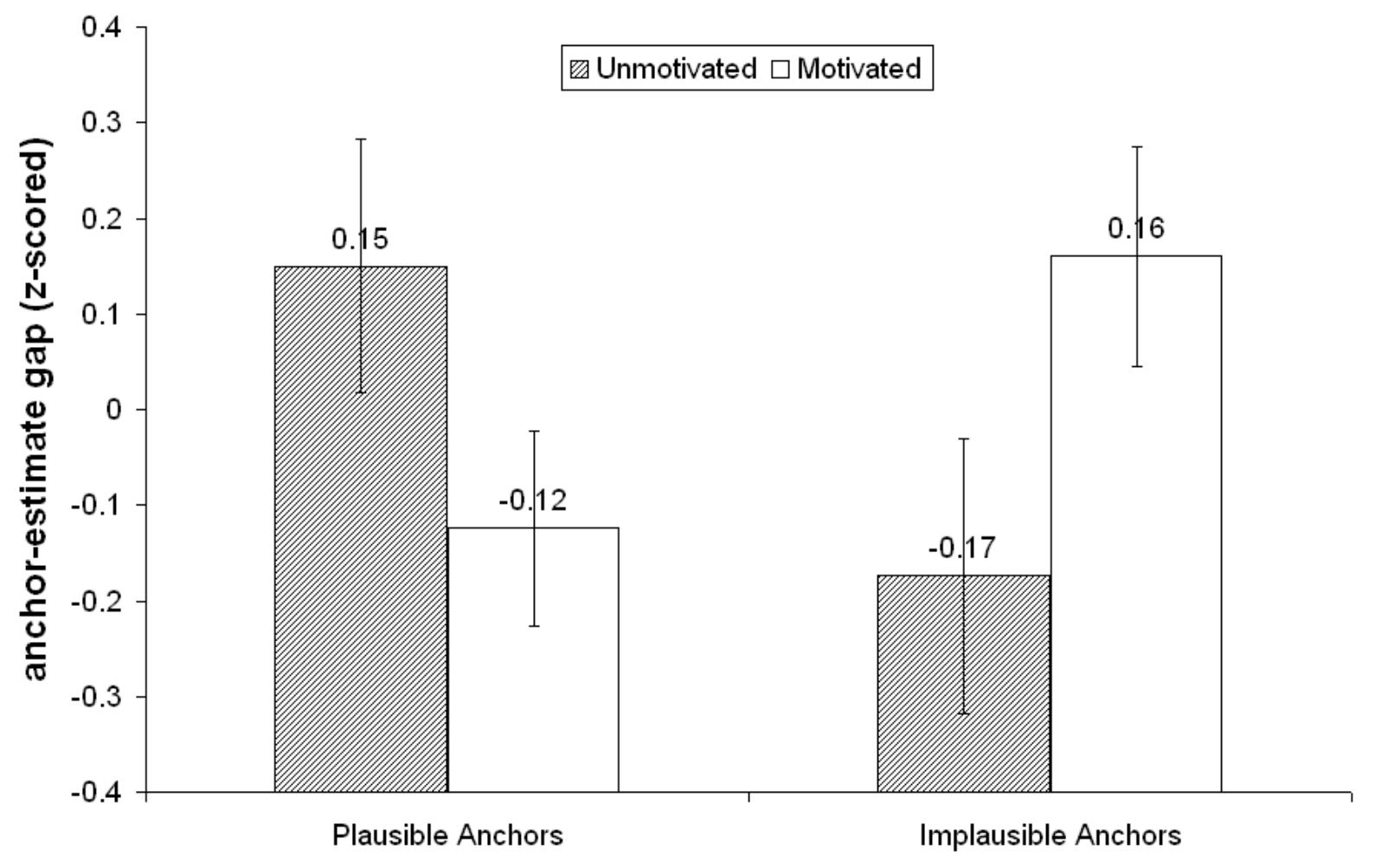

